\title{
Out-of-Plane Static Analysis of Nanoarches Using Eringen's Nonlocal Elasticity Theory
}

\author{
Serhan Aydin Aya, Ekrem Tufekci \\ Istanbul Technical University, \\ Faculty of Mechanical Engineering, Gumussuyu, 34437, Istanbul, Turkey \\ ayas@itu.edu.tr; tufekcie@itu.edu.tr
}

\begin{abstract}
Out-of-plane static behavior of circular nanobeams with point loads are investigated. Inclusion of small length scales such as lattice spacing between atoms, surface properties, grain size etc. are considered in the analysis by employing Eringen's nonlocal elasticity theory in the formulations. The nonlocal equations are arranged in cylindrical coordinates and applied to the beam theory. The effect of shear deformation is considered. The governing differential equations are solved exactly by using the initial value method. The displacements, rotation angle about the normal and tangential axes and the force resultants are established.
\end{abstract}

Keywords: Nanoarches, nonlocal elasticity, out-of-plane statics, exact solution, initial value method

\section{Introduction}

Nanotechnology has been rapidly growing over the last two decades. Thus, the utilization of micro/nanostructures in engineering applications and analysis of mechanical behavior of these structures is currently research subject of many studies. To design novel nano-devices, it is essential to have rigorous understanding of their physical properties. Nanobeams are widely used as a structural element for nanodevices and nanomachines. The development of nano-sized machines depends on proper understanding of mechanical behavior of these nano-sized beam elements. Small length scales such as lattice spacing between atoms, surface properties, grain size etc. are need to be considered when applying any classical continuum model. Couple-stress theory is a size-dependent continuum mechanics model for the analysis of nanostructures which uses virtual work and kinematical assumptions to explain the skew-symmetric nature of the couplestress tensor and shows that mean curvature is in fact the correct energy conjugate measure of deformation [3]. This theory is applied for the static bending and free vibration problems of a simply supported curved beam [4] and it is concluded that the predicted trends confirm the size effect at the micron scale observed in the experiments. Berrabah et al. [5] proposed a unified nonlocal shear deformation theory to study the bending, buckling and free vibration of nanobeams. Both small scale effect and transverse shear deformation effect were considered in the model and Hamilton's principle was used for obtaining equations of motion and analytical solutions were presented for the deflection, buckling load, and natural frequency of a simply supported nanobeam.

The nonlocal theory of elasticity, initiated by Eringen and Edelen [6], which takes the small scale effect into account, has been widely used for the analysis of nanostructures. This theory states that the stress at a given reference point of a body is a function of the strain field at every point in the body; hence, the theory takes the long range forces between atoms and the scale effect into account in the formulation.

This theory is widely used but their application to problems formulated in curvilinear coordinates is scarce. Motivated by this fact, a new nonlocal beam theory within the framework of Eringen's nonlocal elasticity theory for circular nanobeams under mechanical loadings is presented. The objective of the paper is to derive the nonlocal equations for out-of-plane static behavior of curved nanobeam. In order to achieve this goal, the equations of nonlocal elasticity theory are written in cylindrical coordinate system and then implemented in the beam theory. Shear deformation effect and its size-dependent effects along with the size-dependent effects of bending and torsional moments are incorporated in the analytical model. Based on the initial value method, the exact analytical solution of the governing differential equations is obtained. The equations provide sufficient generality in the choice of loading and boundary conditions. Main contribution of this study is to give the exact analytical solutions for the circular curved beams with uniform cross-section. The effectiveness of the model is investigated by solving several examples. The analytical expressions for displacement and 
rotation are given explicitly for clamped-free nanobeams with a tip load. The clamped-clamped circular nanobeam loaded with a force at any $\theta_{K}$ coordinate is also investigated. The effects of the loading type and small scale parameter on the displacements, rotation angle about the binormal axis and the force resultants are analyzed. The numerical results reveal that the deformation of the beam predicted by the nonlocal model are larger than those by the classical curved beam models. The differences in the deformation predicted by both theories decrease with the increase of the small scale parameter.

\section{Analysis}

Response of materials at the nanoscale is different from those of their bulk counterparts. Nonlocal elasticity is first considered by Eringen [6]. It is assumed that the stress at a reference point is a function of the strain at every point of the continuum. In isotropic media, Eringen [7] assumed that a unique kernel weights all entries of stiffness tensor equally and proposed a differential form of the nonlocal relation as:

$$
\sigma_{i j}^{n l}(\boldsymbol{x})=\int_{\Omega} \alpha\left(\boldsymbol{x}, \boldsymbol{x}^{\prime}\right) \sigma_{i j}^{l}\left(\boldsymbol{x}^{\prime}\right) d \Omega
$$

where $\sigma_{i j}^{n l}$ and $\sigma_{i j}^{l}$ are nonlocal and local stress tensors, respectively, $\boldsymbol{x}$ and $\boldsymbol{x}^{\prime}$ are position vectors for two material points in domain $\Omega$ and $\alpha$ is a scalar kernel function. The integral equations of nonlocal elasticity can be simplified to an equivalent partial differential equation by making certain assumptions [7]:

$$
\left(1-\gamma^{2} \nabla^{2}\right) \boldsymbol{\sigma}^{n l}=\boldsymbol{\sigma}^{l}
$$

where $\nabla^{2}$ is the Laplacian operator, $\boldsymbol{\sigma}^{n l}$ and $\boldsymbol{\sigma}^{l}$ are the nonlocal and local stress tensors, respectively, and $\gamma=e_{0} a$ is the nonlocal parameter that describes the effect of small scale on the mechanical behavior. The parameter $e_{0}$ is a constant which has to be determined for each material independently and $a$ is an internal characteristics length. Eringen [7] estimated the parameter $e_{0}$ as 0.39. Several authors reported that the value of $e_{0} a$ varies between 0 to $2 \mathrm{~nm}$ for analyzing carbon nanotubes [8,9].

The expressions for components of Laplacian of the symmetrical second order tensor are given by Povstenko [10]. Using these equations, the Laplacian of the nonlocal stress tensor $\boldsymbol{\sigma}^{n l}$ in cylindrical coordinates are obtained as follows:

$$
\begin{gathered}
\left(\nabla^{2} \boldsymbol{\sigma}^{n l}\right)_{r r}=\nabla^{2} \sigma_{r r}^{n l}-\frac{4}{r^{2}} \frac{\partial \sigma_{r \theta}^{n l}}{\partial \theta}-\frac{2}{r^{2}}\left(\sigma_{r r}^{n l}-\sigma_{\theta \theta}^{n l}\right) \\
\left(\nabla^{2} \boldsymbol{\sigma}^{n l}\right)_{r r}=\nabla^{2} \sigma_{r r}^{n l}-\frac{4}{r^{2}} \frac{\partial \sigma_{r \theta}^{n l}}{\partial \theta}-\frac{2}{r^{2}}\left(\sigma_{r r}^{n l}-\sigma_{\theta \theta}^{n l}\right) \\
\left(\nabla^{2} \boldsymbol{\sigma}^{n l}\right)_{r \theta}=\nabla^{2} \sigma_{r \theta}^{n l}-\frac{4}{r^{2}} \sigma_{r \theta}^{n l}+\frac{2}{r^{2}} \frac{\partial}{\partial \theta}\left(\sigma_{r r}^{n l}-\sigma_{\theta \theta}^{n l}\right) \\
\left(\nabla^{2} \boldsymbol{\sigma}^{n l}\right)_{r z}=\nabla^{2} \sigma_{r z}^{n l}-\frac{1}{r^{2}} \sigma_{r z}^{n l}-\frac{2}{r^{2}} \frac{\partial \sigma_{\theta z}^{n l}}{\partial \theta} \\
\left(\nabla^{2} \boldsymbol{\sigma}^{n l}\right)_{\theta z}=\nabla^{2} \sigma_{\theta z}^{n l}-\frac{1}{r^{2}} \sigma_{\theta z}^{n l}+\frac{2}{r^{2}} \frac{\partial \sigma_{r z}^{n l}}{\partial \theta} \\
\left(\nabla^{2} \boldsymbol{\sigma}^{n l}\right)_{z z}=\nabla^{2} \sigma_{z z}^{n l}
\end{gathered}
$$

where,

$$
\nabla^{2} f=\frac{\partial^{2} f}{\partial r^{2}}+\frac{1}{r} \frac{\partial f}{\partial r}+\frac{1}{r^{2}} \frac{\partial^{2} f}{\partial \theta^{2}}+\frac{\partial^{2} f}{\partial z^{2}}
$$

The formulation of problems of curved beams is given in the Frenet coordinate system. The cylindrical and Frenet coordinate systems are given in Fig. 1. The stresses in Frenet coordinate system are given as $\sigma_{t}, \sigma_{n t}$ and $\sigma_{t b}$. The remaining stresses $\left(\sigma_{n}, \sigma_{b}\right.$ and $\left.\sigma_{n b}\right)$ are assumed as zero. For out-of-plane problems, the stresses $\sigma_{t}, \sigma_{n t}$ and $\sigma_{t b}$ and stress 
resultants $F_{b}^{n l}, M_{n}^{n l}$ and $M_{t}^{n l}$ are considered. The relations between the stresses in Frenet and cylindrical coordinates are as follows:

$$
\begin{aligned}
& \sigma_{n t}=-\sigma_{r \theta} \neq 0 ; \sigma_{t b}=\sigma_{\theta z} \neq 0 ; \sigma_{t}=\sigma_{\theta \theta} \neq 0 \\
& \sigma_{n}=-\sigma_{r r}=0 ; \sigma_{b}=\sigma_{z z}=0 ; \sigma_{n b}=-\sigma_{r z}=0
\end{aligned}
$$

A uniform circular beam is considered in this study, thus, the radius of the beam is constant, i.e. $R(\theta)=R$, and the coordinate $r$ is described as (Fig. 1);

$$
r=R+\bar{r} \quad \partial r=\partial \bar{r}
$$

It is assumed that $\bar{r} / R \ll 1$ (beam assumption).

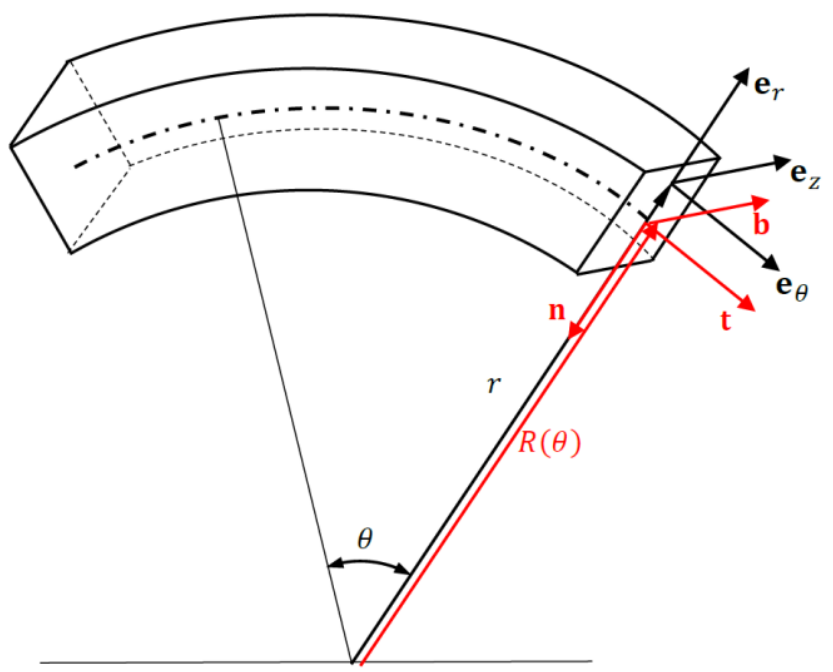

Fig. 1: The Frenet and cylindrical coordinates of a curved beam.

After that, the governing differential equations of nonlocal beams can be written in the following form;

$$
\begin{gathered}
\frac{d v(\theta)}{d \theta}=-R \Omega_{n}(\theta)+\frac{k_{b} R}{G A}\left(1+\frac{\gamma^{2}}{R^{2}}\right) F_{b}^{n l} \\
\frac{d \Omega_{n}(\theta)}{d \theta}=-\Omega_{t}(\theta)+\frac{R}{E I_{n}}\left(1+\frac{\gamma^{2}}{R^{2}}\right) M_{n}(\theta) \\
\frac{d \Omega_{t}(\theta)}{d \theta}=\Omega_{n}(\theta)+\frac{R}{G J}\left(1+\frac{3 \gamma^{2}}{2 R^{2}}\right) M_{t}^{n l} \\
\frac{d M_{n}^{n l}(\theta)}{d \theta}=-M_{t}^{n l}(\theta)+R F_{b}^{n l} \\
\frac{d M_{t}^{n l}(\theta)}{d \theta}=M_{n}^{n l}(\theta) \\
\frac{d F_{b}^{n l}(\theta)}{d \theta}=0
\end{gathered}
$$

These equations can also be stated in the matrix form as:

$$
\frac{d \mathbf{y}(\theta)}{d \theta}=\mathbf{A}(\theta) \mathbf{y}(\theta)
$$


where $\mathbf{y}$ is the vector of variables, namely, $v, \Omega_{n}, \Omega_{t}, M_{n}^{n l}, M_{t}^{n l}, F_{b}^{n l} ; \mathbf{A}(\theta)$ is the $6 \times 6$ coefficient matrix. In this study, the components of the coefficient matrix are constant. The solution of the homogeneous system in Eqn. (19) can be expressed as:

$$
\mathbf{y}(\theta)=\mathbf{Y}\left(\theta, \theta_{0}\right) \mathbf{y}_{0}
$$

or more explicitly:

$$
\left[\begin{array}{c}
v(\theta) \\
\Omega_{n}(\theta) \\
\Omega_{t}(\theta) \\
M_{n}^{n l}(\theta) \\
M_{t}^{n l}(\theta) \\
F_{b}^{n l}(\theta)
\end{array}\right]=\left[\begin{array}{llllll}
Y_{11} & Y_{12} & Y_{13} & Y_{14} & Y_{15} & Y_{16} \\
Y_{21} & Y_{22} & Y_{23} & Y_{24} & Y_{25} & Y_{26} \\
Y_{31} & Y_{32} & Y_{33} & Y_{34} & Y_{35} & Y_{36} \\
Y_{41} & Y_{42} & Y_{43} & Y_{44} & Y_{45} & Y_{46} \\
Y_{51} & Y_{52} & Y_{53} & Y_{54} & Y_{55} & Y_{56} \\
Y_{61} & Y_{62} & Y_{63} & Y_{64} & Y_{65} & Y_{66}
\end{array}\right]\left[\begin{array}{c}
v_{0} \\
\Omega_{n 0} \\
\Omega_{t 0} \\
M_{n 0} \\
M_{t 0} \\
F_{b 0}
\end{array}\right]
$$

where $\mathbf{Y}\left(\theta, \theta_{0}\right)$ is the fundamental matrix which is obtained from the solution of the system of homogeneous equations, and $\mathbf{y}_{0}=\mathbf{y}\left(\theta_{0}\right)$ is the vector of initial values at the coordinate $\theta_{0}$, (in this study $\theta_{0}=0$ ). The fundamental matrix satisfies the following requirements:

$$
\begin{array}{cc}
\mathbf{Y}\left(\theta_{1}, \theta_{2}\right) \mathbf{Y}\left(\theta_{2}, \theta_{3}\right)=\mathbf{Y}\left(\theta_{1}, \theta_{3}\right), & \mathbf{Y}\left(\theta_{1}, \theta_{2}\right)=\mathbf{Y}^{-1}\left(\theta_{2}, \theta_{1}\right) \\
\frac{d \mathbf{Y}\left(\theta, \theta_{0}\right)}{d \theta}=\mathbf{A}(\theta) \mathbf{Y}\left(\theta, \theta_{0}\right), & \mathbf{Y}\left(\theta_{0}, \theta_{0}\right)=\mathbf{I}
\end{array}
$$

where $\mathbf{I}$ is unit matrix.

If the initial values, $v_{0}, \Omega_{n 0}, \Omega_{t 0}, M_{n 0}^{n l}, M_{t 0}^{n l}, F_{b 0}^{n l}$ are known, the solution given in Eqn. (20) can be obtained analytically. These values can be solved from a system of linear equations which are obtained from the boundary conditions of the beam.

In this study, as a general case, a circular uniform beam with point loads at the coordinate $\left(\theta=\theta_{K}\right)$ is also investigated. This type of beam has two regions and the solutions for both regions are knows as:

$$
\begin{array}{llr}
\mathbf{y}_{1}\left(\theta_{1}\right)=\mathbf{Y}\left(\theta_{1}, \theta_{0}\right) \mathbf{y}_{10} & \text { for } & -\theta_{A} \leq \theta_{1} \leq \theta_{K} \\
\mathbf{y}_{2}\left(\theta_{2}\right)=\mathbf{Y}\left(\theta_{2}, \theta_{K}\right) \mathbf{y}_{2 K} & \text { for } & \theta_{K} \leq \theta_{1} \leq \theta_{B}
\end{array}
$$

where $\mathbf{y}_{2 K}$ is the vector of initial values for the second region at coordinate $\theta_{K}$. In order to calculate twelve components of the vectors $\mathbf{y}_{10}$ and $\mathbf{y}_{2 K}$, twelve equations of boundary and continuity conditions are used. The continuity condition at that point is:

$$
\mathbf{y}_{1}\left(\theta_{K}\right)+\mathbf{K}=\mathbf{y}_{2 K}
$$

where $\mathbf{K}=\left[\begin{array}{llllll}0 & 0 & 0 & M_{K n} & M_{K t} & F_{K b}\end{array}\right]^{\mathrm{T}}$ is the loading vector. Thus, Eqn. (24) is rewritten as:

$$
\mathbf{y}_{2}\left(\theta_{2}\right)=\mathbf{Y}\left(\theta_{2}, \theta_{K}\right) \mathbf{y}_{1}\left(\theta_{K}\right)+\mathbf{Y}\left(\theta_{2}, \theta_{K}\right) \mathbf{K}
$$

By substituting Eqn. (23) into Eqn. (26), the following equation is obtained:

$$
\mathbf{y}_{2}\left(\theta_{2}\right)=\mathbf{Y}\left(\theta_{2}, \theta_{K}\right) \mathbf{Y}\left(\theta_{K}, \theta_{0}\right) \mathbf{y}_{10}+\mathbf{Y}\left(\theta_{2}, \theta_{K}\right) \mathbf{K}
$$

Eqn. (27) can be rewritten by using Eqn. (22) as follows:

$$
\mathbf{y}_{2}\left(\theta_{2}\right)=\mathbf{Y}\left(\theta_{2}, \theta_{0}\right) \mathbf{y}_{10}+\mathbf{Y}\left(\theta_{2}, \theta_{0}\right) \mathbf{Y}^{-1}\left(\theta_{K}, \theta_{0}\right) \mathbf{K}
$$


Then, the analytical functions of the displacements, rotation angle of the cross-section and the force resultants for both region can be obtained.

\section{A circular nanobeam with a point load $F_{b K}$ at an arbitrary coordinate $\theta_{K}$}

In this example, a clamped-clamped circular nanobeam with a concentrated unit load $F_{b K}$ in binormal direction at coordinate $\theta_{K}=30^{\circ}$ is considered (Fig. 2). The beam has an opening angle $\theta_{T}=2 \pi / 3$, slenderness ratio $\lambda=100$ and small scale parameter $R / \gamma=1$. The displacement, rotation angle of cross section about normal and tangential axes, and force resultants for unsymmetrical loading condition are investigated.

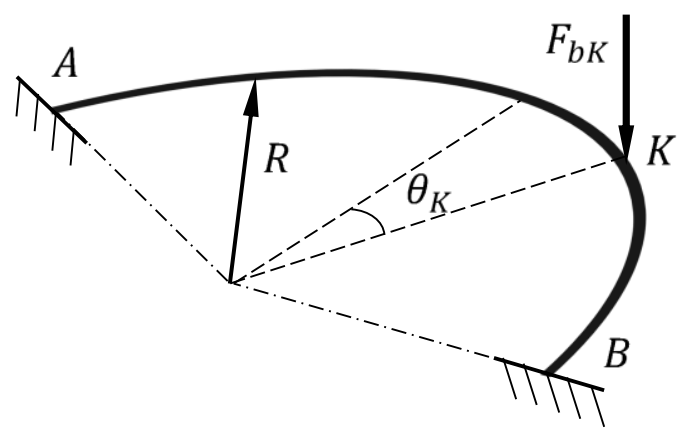

Fig. 2: Clamped-clamped circular nano beam with a point load $F_{b K}$ at an arbitrary coordinate $\theta_{K}\left(\theta_{T}=2 \pi / 3\right)$.

The shear deformation effect is included in the calculations. The results of nonlocal elasticity and classical (local) theory are given in Figs. 3-5.

Figs. 4 and 5 present the rotation angle of the cross-section about normal and tangential axes, for the circular nanobeam obtained from both theories. The results of the classical (local) theory and those of nonlocal theory are significantly different.

It is observed that the result of the nonlocal theory always larger than that by the classical model. The difference between the results of both theories decreases, if the small scale parameter $R / \gamma$ increases. Thus, the small scale parameter has a relevant importance in calculation of displacement and rotation of the cross-section.

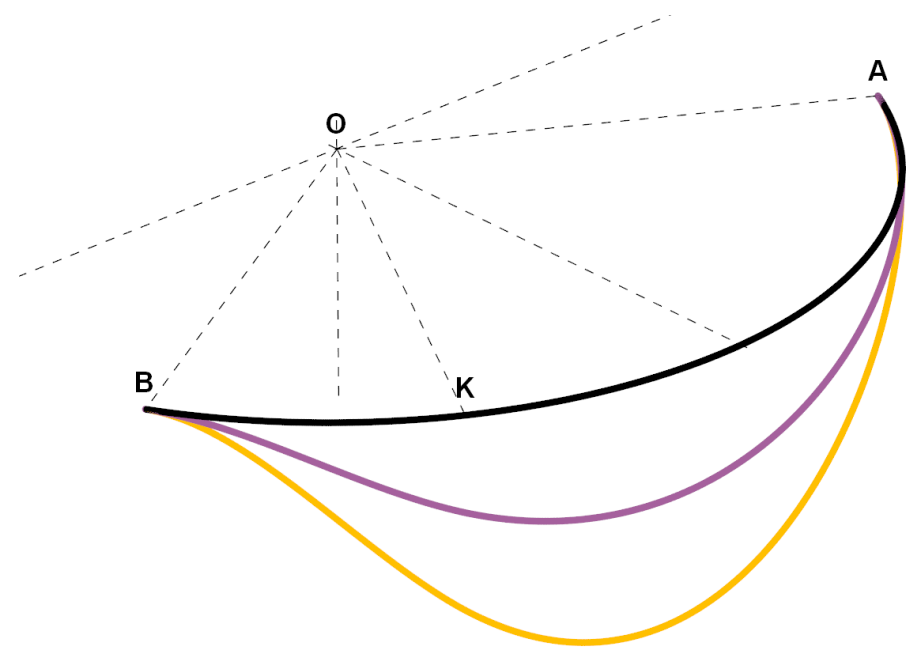

Fig. 3: Displacements in binormal direction $(v)$ obtained by local and nonlocal theories ( — Underformed Axis is, — Nonlocal, All Effect — Local, All Effects 


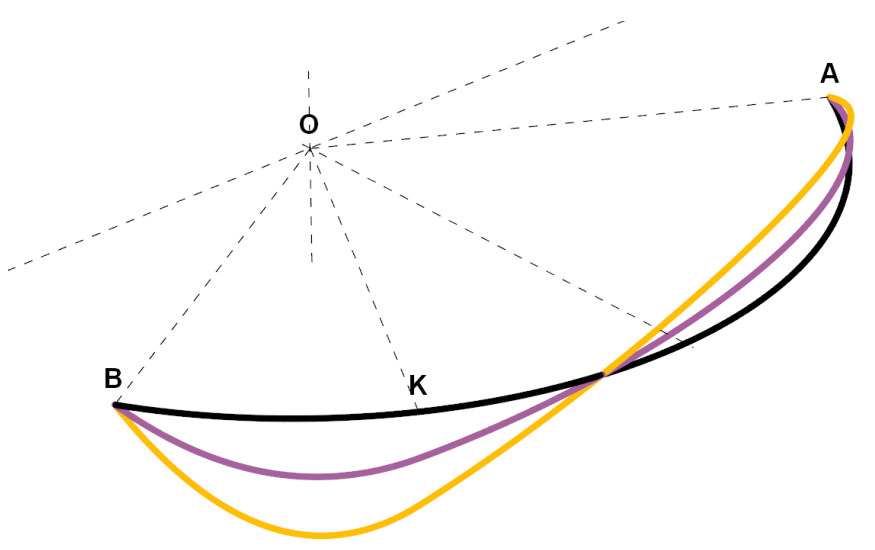

Fig. 4: Rotation angle of the cross-section about normal axis $\left(\Omega_{n}\right)$ obtained by local and nonlocal theories

( — Underformed Axis is, — Nonlocal, All Effect — Local, All Effects

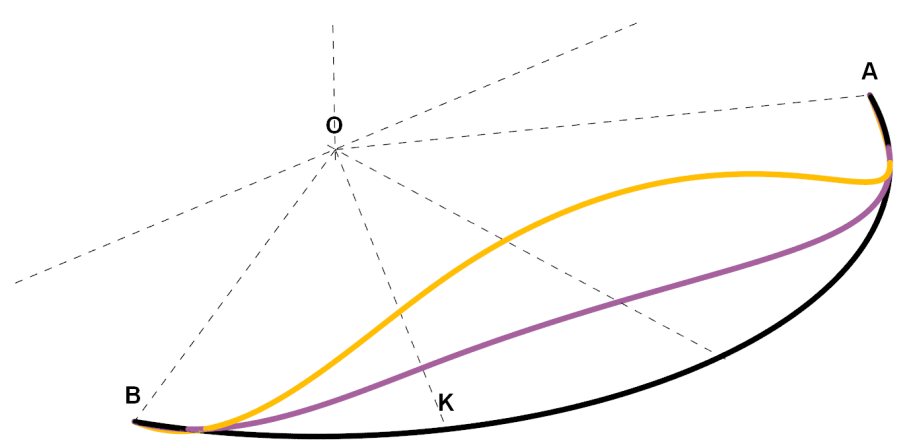

Fig. 5: Rotation angle of the cross-section about tangential axis $\left(\Omega_{t}\right)$ obtained by local and nonlocal theories ( - Underformed Axis is, — Nonlocal, All Effect — Local, All Effects

The force resultants, i.e. bending moment in normal direction $M_{n}$, torsional moment in tangential direction $M_{t}$ and shear force in binormal direction $F_{b}$ obtained from classical and nonlocal theories are also investigated. Both of the classical (local) theory and the nonlocal theory give almost same results. The diagrams are not given for the brevity.

\section{Conclusion}

To the best knowledge of the authors, the exact analytical solution of the out-of-plane static problems of circular nanobeams is the first time in the literature. The results indicate that this general nonlocal circular beam theory can be used easily for a parametric study investigating the effects of loading types, geometric properties (opening angle, slenderness ratio, small scale parameter). Although only uniform circular nanobeams bearing concentrated loads are investigated in this paper, the equations can easily be expanded to provide sufficient generality in the choice of loading (varying distributed load) and geometry (varying curvature and cross-section). It is also possible to expand the formulation given in this study for investigating the dynamics of curved nanobeams. Another possible application is to develop an exact nonlocal beam finite element for practical engineering purposes. It is expected that this study would be very helpful in design of curved beam components in MEMS and NEMS applications, particularly whose main duties are to bear securely the applied forces. The results of this study may be used as benchmarks for other theoretical or numerical methods afterwards.

\section{Acknowledgements} $112 \mathrm{M} 404$.

This study has been supported by The Scientific and Technological Council of Turkey (TUBITAK) with Project No: 


\section{References}

[1] D. Srivastava and C. Wei, "Nanomechanics of carbon nanotubes and composites," Appl. Mech. Rev., vol. 56, pp. 215230, 2003.

[2] B. Arash, J. W. Jiang and T. Rabczuk, "A review on nanomechanical resonators and their applications in sensors and molecular transportation," Appl. Phys. Rev., vol. 2, pp. 021301 2-21, 2015.

[3] A. R. Hadjesfandiari and G. F. Dargush, "Couple stress theory for solids," Int. J. Solids Struct., vol. 48, pp. 1499$1512,2011$.

[4] Y. P. Liu and J. N. Reddy, "A Nonlocal Curved Beam Model Based on a Modified Couple Stress Theory," Int. J. Struct. Stab. Dy., vol. 11, pp. 495-512, 2011.

[5] H.M. Berrabah, A. Tounsi, A. Semmah and E. A. A. Bedia, "Comparison of various refined nonlocal beam theories for bending, vibration and buckling analysis of nanobeams," Struct. Eng. Mech., vol. 48, pp. 351-365, 2013.

[6] A. C. Eringen and D. G. B. Edelen, "Nonlocal elasticity," Int. J. Eng. Sci., vol. 10, pp. 233-248, 1972.

[7] A. C. Eringen, "On differential equations of nonlocal elasticity and solutions of screw dislocation and surface waves," J. Appl. Phys., vol. 54, pp. 4703-4710, 1983.

[8] L. J. Sudak, "Column buckling of multi-walled carbon nanotubes using nonlocal elasticity," J. Appl. Phys., vol. 94, pp. 7281-7287, 2003.

[9] L. F. Wang and H. Y. Hu, "Flexural wave propagation in single-walled carbon nanotubes," Phys. Rev. B, vol. 71, pp. 195412 1-7, 2003.

[10] Y. Z. Povstenko, "Straight disclinations in nonlocal elasticity,” Int. J. Eng. Sci., vol. 33, pp. 575-582, 1995. 